\title{
SESSION-RPE IS A VALUABLE INTERNAL LOAD EVALUATION METHOD IN BEACH VOLLEYBALL FOR BOTH GENDERS, ELITE AND AMATEUR PLAYERS, CONDITIONING AND TECHNICAL SESSIONS, BUT LIMITED FOR TACTICAL TRAINING AND GAMES
}

\author{
Corrado Lupo, Alexandru Nicolae Ungureanu, and Paolo Riccardo Brustio \\ Neuro Muscular Function Research Group, School of Exercise and Sport (SUISM), \\ Department of Medical Sciences, University of Turin, Italy
}

Original scientific paper

DOI: $10.26582 / \mathrm{k} .52 .1 .4$

\begin{abstract}
:
This study aimed to verify the validity of session-RPE method to monitor the internal training load (ITL) in beach volleyball players by considering sessions related to different genders, competition levels (elite or amateur), and types of session (conditioning, technical, or tactical/game). Session-RPE and Edwards' methods were applied to quantify the ITLs of 12 elite (18 players; 197 individual sessions) and 12 amateur (18 players; 189 individual sessions) training sessions. Very large relationships between the two methods emerged for both competition level (Elite: $r=.77$; Amateur: $r=.75$ ) and gender (male: $r=.76$; female: $r=.75$ ) subgroups, and conditioning sessions $(\mathrm{r}=.75)$. Large relationships emerged for technical $(\mathrm{r}=.61)$ sessions, whereas tactical/ game sessions resulted only in moderate relationships $(\mathrm{r}=.36)$. Beach volleyball coaches could adequately use session-RPE method to monitor training for players of different genders, competition levels, and types of session, although tactical/game sessions should be considered with some caution.
\end{abstract}

Key words: rating of perceived exertion, heart rate, internal training load, situational sports, training monitoring

\section{Introduction}

Subjective ratings of perceived exertion (RPE) have demonstrated to be a practical, valuable, and inexpensive tool for quantifying athletes' internal training load (ITL) (Borg, 1998; Foster, et al., 1995). Based on the understanding that athletes can naturally monitor the physiological stress their bodies experience during exercise, RPE has been assessed using category-ratio (Borg, 1998; Foster, et al., 1995). As a consequence, several authors have successfully verified the entity of ITL by multiplying the athlete's RPE for total duration (expressed in minutes) of a training session (i.e., session-RPE) in steady-state (Herman, Foster, Maher, Mikat, \& Porcari, 2006) and non-steady-state (Foster, et al., 1995) exercises, in endurance sports (Foster, 1998), as well as in individual (Minganti, Capranica, Meeusen, \& Piacentini, 2011; Wallace, Slattery, \& Coutts, 2009) and team sports (Coutts, Rampinini, Marcora, Castagna, \& Impellizzeri, 2009; Coutts, Vitasovic Gomes, Viveiros, \& Aoki, 2010; Lupo, Tessitore, Gasperi, \& Gómez, 2017b).
Although the monitoring of ITL has been studied in several training conditions, some limitations were inferred for situational disciplines because of their complexity related to different goals and types of exercise. In addition, according to Impellizzeri, Rampinini, Coutts, Sassi, and Marcora (2004), team sports can be characterized by intermittent exercises relying on both the aerobic and anaerobic energy sources (Bangsbo, 2000), thus allowing for speculation on a higher occurrence of heterogeneous RPE values (and variable relationships with measurements of reference such as HR) for this sport category in comparison to individual ones (Foster, 1998). Nevertheless, satisfactory findings on session-RPE validity were recently provided also for invasion team sports (Coutts, et al., 2009; Lupo, Capranica, \& Tessitore, 2014; Lupo, et al., 2017b) and other situational disciplines (Casolino, et al., 2012; Lupo, et al., 2017a).

However, only few studies (Aoki, et al., 2017; Coutts, et al., 2010; Murphy, Duffield, Kellett, $\&$ Reid, 2016) on the session-RPE validity were 
focused on return and territorial (i.e., not invasion) sports, also reporting controversial results. In particular, a study focused on volleyball (Aoki, et al., 2017) aimed to describe and compare the training periodization of two age-categories (U16 versus U19), verifying the effect of the periodized training program on ITL, mood states, and vertical jump performance of young players. Even though session-RPE (with the Borg's category ratio 10 RPE scale; CR-10) was applied to quantify the athletes' ITL, no measurement of reference had been considered to establish the validity of this method for this particular team sport. Similarly, no result regarding the session-RPE validity has been provided for a study on elite tennis (Coutts, et al., 2010). Conversely, a high correlation between external and ITLs was provided in a study on youth tennis players (Murphy, et al., 2016) performing 259 individual drills, where session-RPE values actually reported a partial correlation with the ITLs determined by the HR-based method. Therefore, the promotion of further studies on the correlation between session-RPE and HR-based methods could contribute to better clarify the validity of the first ITL quantification.

Although session-RPE has been widely studied in team sports, demonstrating to be a valuable tool in general, relevant differences have also been reported for the same sport and sample of athletes in relation to different type of training sessions (Lupo, et al., 2014, 2017a,b). As a consequence, different training modalities characterizing return (i.e., volleyball, tennis, beach volleyball) and invasion (i.e., soccer, basketball, water polo) sports could determine different grades of correlation between session-RPE and Edwards' methods.

Among return sports, beach volleyball represents a worldwide growing sport at amateur and elite level, especially from its first inclusion in the program of the Olympic Games (Atlanta 1996). This game is played by two teams with two players each one, on an 8x16 m court, and currently characterized by the scoring system with two 21-point sets without the need to have the service to score, and an eventual 15-point third set in case of an equal set score. In addition, the intermittent high-intensity actions with rallies lasting less than seven seconds on average as well as the fact that around one quarter of match time is spent in active play (Pilaczyńska-Szcześniak, et al., 2011) highlight the presumed complexity of quantifying the players' ITLs in this team sport. Therefore, especially for the last two performance aspects, the monitoring of ITL in beach volleyball players appears as a necessity if we want to get a deeper insight into players' ITL not provided by simple inferences from the external training load parameters (i.e., duration of workouts, occurrences of technical activity repetitions, etc).
Although in the studies on session-RPE involving the participation of male and female athletes data were usually merged (Minganti, et al., 2011; Murphy, et al., 2016; Wallace, et al., 2016), a separate gender analysis of players' ITL could made results more consistent. At the same time, an eventual gender difference in the correlation between the HR-based method and session-RPE could be better explained by other factors such as competition level, thus highlighting the need to consider also the latter factor. Actually, the validity of session-RPE was already discriminated in terms of competition level in a study on small-sided soccer games (Coutts, et al., 2009), but the different nature of return and invasion team sports highlights the need of providing more specific findings on this issue.

Therefore, the aims of this study were to verify the grade of correlation between session-RPE and Edwards' methods in beach volleyball players by considering sessions associated to different genders, competition levels (elite or amateur), and types of session (conditioning, technical, or tactical/game sessions), as well as to sessions performed by each individual player.

\section{Methods}

\section{Design}

In line with previous studies (Coutts, et al., 2009, 2010; Impellizzeri, et al., 2004), the validity of session-RPE method in beach volleyball has been established according to its correlation to the Edwards' method (Edwards, 1993). In particular, for the lattert method, ITL is determined by summing up the scores obtained by the multiplication of the accumulated time (in minutes) spent in every of the five HR zones (related to the individual HRmax) with the corresponding coefficients (i.e., $50-60 \%=1$; $60-70 \%=2 ; 70-80 \%=3 ; 80-90 \%=4 ; 90-100 \%=5$ ). For this study, no maximal intensity incremental test was performed to provide the actual players' HRmax. Instead, the beach volleyball players' HRmax was estimated according to the theoretical "220 - age" formula (Fox III \& Naughton, 1972), therefore no assurance of recognizing this reference according to the HR peak performed during training has been guaranteed.

For the session-RPE method, players used to indicate their self-evaluation of RPE after each training session by means of the Borg CR-10 scale modified by Foster et al. (1995), which is a categoryratio scale characterized by scores ranging from a minimum of 0 to a maximum of 10 , with verbal links being: 0="Rest"; 1="Very, Very Easy"; 2="Easy"; 3="Moderate"; 4="Somewhat Hard"; 5="Hard"; 7="Very Hard"; 10="Maximal". Beach volleyball players were familiarized with the CR-10 scale two weeks prior to the start of the experimental period. 
In this preliminary period, players took special care about the meaning of scores according to the verbal links attributed to the whole training session (and not only its last phase) to correctly quantify ITL.

All amateur and elite training sessions were monitored during five weeks of the pre-season period of the 2014/15 Campionato Italiano Assoluto di Beach Volley. All training sessions were scheduled in afternoons, on Mondays, Wednesdays, and Fridays, at an indoor beach volleyball court. Each single session has been classified by the main portion of its contents (i.e., at least $40 \%$ of the total session duration, 10-20-minute warm-up excluded) into either the conditioning, technical, or tactical/ game session.

\section{Participants}

The institutional review board approved this study and an informed consent form, regarding the potential risks and benefits associated with the participation, has been obtained from 18 elite (nine men: age $=23 \pm 1$ years, body height $=188 \pm 5 \mathrm{~cm}$, body mass $=78 \pm 7 \mathrm{~kg}$; nine women: age $=22 \pm 3$ years, body height $=181 \pm 6 \mathrm{~cm}$, body mass $=69 \pm 9 \mathrm{~kg})$ and 18 amateur (nine men: age $=28 \pm 4$ years, body height $=179 \pm 12 \mathrm{~cm}$, body mass $=77 \pm 9 \mathrm{~kg}$; nine women: age $=26 \pm 4$ years, body height $=172 \pm 6 \mathrm{~cm}$, body mass $=64 \pm 8 \mathrm{~kg}$ ) players. Elite beach volleyball players had to fulfill the following two inclusion criteria: to compete at the 2014/15 Campionato Italiano Assoluto di Beach Volley (i.e., the highest national beach volleyball championship in Italy), and to have at least seven years of beach volleyball training experience (consisting of a minimum of three to a maximum of seven 90-180-min training weekly sessions); conversely, no particular competition and training background was considered as the inclusion criteria for the amateur players.

\section{Procedures}

\section{Edwards' HR-based method}

During each training session, individual HRs were recorded every one second by means of HR monitors (Polar Team System, Polar, Kempele, Finland) with a transmitter belt (consisting also of an internal memory) placed on the beach volleyball player's chest before each training session. Then, to establish individual ITLs, HR data were transferred onto a computer and classified according to the Edwards' method (Edwards, 1993).

\section{Session-RPE method}

To assess the session-RPE in beach volleyball players, the total duration of a session was registered, and the Italian translation of the CR-10 scale modified by Foster et al. (1995) was submitted to players around 30 minutes after the end of each training session. Then, the individual CR-10 RPE scores of each training session were multiplied by the correspondent duration (minutes) to calculate the related session-RPE (Foster, et al., 1995).

\section{Statistical analyses}

Means and standard deviations were calculated for each analyzed variable. The statistical analyses were performed using the statistical software IBM SPSS Statistics for Windows (Version 21.0., Armonk, NY: IBM Corp) and the criterion for significance was set at $\mathrm{p} \leq .05$. Before using parametric statistical test procedures, the assumptions of normality and sphericity were verified by Shapiro-Wilk normality test and Mauchly's test, respectively.

A 2 Gender (i.e., male, female) X 2 Competition levels (i.e., elite, amateur) X 3 Types of session (i.e., conditioning, technical, tactical/game) ANOVA for repeated measure was applied to ascertain differences for Edwards' ITLs, considering gender and competition level as the between-factor and type of session as the within-factor. In case of significance, the post-hoc analysis was performed with Bonferroni corrections. To provide meaningful analysis for significant comparisons, effect sizes (ESs) were calculated (Cohen, 1998), considering $\leq 0.2,0.6,1.2$, and $>1.2$ as trivial, small, moderate and large ES, respectively (Hopkins, 2008).

The relationships between Edwards' ITL and session-RPE were estimated using the Pearson's product-moment correlation (i.e., r) as well as the coefficient of determination (i.e., $\mathrm{R}^{2}$ ), in relation to gender, competition level, and type of session. Furthermore, small, moderate, large, very large, and nearly perfect correlations were identified with $\mathrm{r}$ values corresponding to $.1, .3, .5, .7$, and .9 , respectively (Hopkins, Marshall, Batterham, \& Hanin, 2009). Finally, the intra-class correlation coefficient (ICC) and the $95 \%$ confidence intervals (95\% C.I.) for the correlation coefficients were calculated.

\section{Results}

One hundred ninety-seven (1:11 $\pm 0: 11, \mathrm{~h}: \mathrm{min})$ and one hundred eighty-nine individual training sessions (0:58 $\pm 0: 06$, h:min) were monitored during 12 elite and 12 amateur team training sessions (9-12 sessions a player in both subcategories), respectively.

No difference was reported for the Edwards' ITLs related to gender and competition level (Table 1), although a strong effect emerged for their interaction $(p<.001, E S$ range $=0.9)$. In particular, for the female beach volleyball players, higher Edwards' ITLs (Table 2) were found in the elite than in the amateur sessions $(\mathrm{p}=.021, \mathrm{ES}=0.9)$, whereas the opposite trend emerged for the male subgroups $(\mathrm{p}=.047, \mathrm{ES}=0.9)$. In addition, higher Edwards' ITLs were found in the elite female sessions than in the elite male ones $(\mathrm{p}=.005, \mathrm{ES}=0.9)$. 
Table 1. Mean and SD (and range) of Edwards, RPE, and session-RPE parameters (Arbitrary Units, AU), and corresponding correlations between session-RPE and Edward's values (ICC, $r, 95 \%$ C.I., $R^{2}$, and $p$ values) of different genders, competition levels(i.e., elite or amateur), and types of session (conditioning, technical, tactical/game)

\begin{tabular}{|c|c|c|c|c|c|c|c|c|c|}
\hline \multirow{2}{*}{$\begin{array}{l}\text { Independent } \\
\text { variables }\end{array}$} & \multirow{2}{*}{ Subgroups } & \multirow{2}{*}{$\begin{array}{l}\text { Edwards } \\
\text { (AU) }\end{array}$} & \multirow{2}{*}{$\begin{array}{l}\text { RPE } \\
\text { (AU) }\end{array}$} & \multirow{2}{*}{$\begin{array}{l}\text { Session-RPE } \\
\text { (AU) }\end{array}$} & \multicolumn{5}{|c|}{$\begin{array}{l}\text { Edwards - session-RPE } \\
\text { correlation indexes }\end{array}$} \\
\hline & & & & & ICC & $r$ & 95\% C.I. & $R^{2}$ & $p$ \\
\hline \multirow{2}{*}{ Gender } & $\begin{array}{l}\text { Female } \\
(n=187)\end{array}$ & $\begin{array}{l}209 \pm 58 \\
(61-324)\end{array}$ & $\begin{array}{c}6.0 \pm 1.6 \\
(3-10)\end{array}$ & $\begin{array}{l}440 \pm 129 \\
(180-720)\end{array}$ & .72 & .75 & $.68-.81$ & .52 & $<.001$ \\
\hline & $\begin{array}{c}\text { Male } \\
(n=204)\end{array}$ & $\begin{array}{c}211 \pm 62 \\
(67-360)\end{array}$ & $\begin{array}{c}5.9 \pm 1.6 \\
(2-9)\end{array}$ & $\begin{array}{l}444 \pm 144 \\
(134-819)\end{array}$ & .71 & .76 & $.69-.81$ & .5 & $<.001$ \\
\hline \multirow{2}{*}{$\begin{array}{l}\text { Competition } \\
\text { level }\end{array}$} & $\begin{array}{c}\text { Elite } \\
(n=197)\end{array}$ & $\begin{array}{l}210 \pm 61 \\
(61-300)\end{array}$ & $\begin{array}{l}5.7 \pm 1.6 \\
(2-10)\end{array}$ & $\begin{array}{c}426 \pm 128 \\
(134-736)\end{array}$ & .75 & .77 & $.71-.82$ & .56 & $<.001$ \\
\hline & $\begin{array}{c}\text { Amateur } \\
(n=194)\end{array}$ & $\begin{array}{c}210 \pm 59 \\
(108-360)\end{array}$ & $\begin{array}{c}6.2 \pm 1.5 \\
(3-10)\end{array}$ & $\begin{array}{l}459 \pm 143 \\
(180-819) \\
\end{array}$ & .69 & .75 & $.68-.81$ & .48 & $<.001$ \\
\hline \multirow{3}{*}{$\begin{array}{l}\text { Type of } \\
\text { session }\end{array}$} & $\begin{array}{l}\text { Conditioning } \\
(n=134)\end{array}$ & $\begin{array}{c}245 \pm 42 \\
(108-360)\end{array}$ & $\begin{array}{c}7.1 \pm 1.3 \\
(4-10)\end{array}$ & $\begin{array}{c}515 \pm 90 \\
(292-720)\end{array}$ & .73 & .75 & $.66-.82$ & .53 & $<.001$ \\
\hline & $\begin{array}{l}\text { Technical } \\
(\mathrm{n}=137)\end{array}$ & $\begin{array}{l}153 \pm 44 \\
(61-285)\end{array}$ & $\begin{array}{c}4.8 \pm 1.1 \\
(2-7)\end{array}$ & $\begin{array}{c}322 \pm 95 \\
(134-665)\end{array}$ & .63 & .61 & $.49-.71$ & .37 & $<.001$ \\
\hline & $\begin{array}{c}\text { Tactical/game } \\
(n=120)\end{array}$ & $\begin{array}{c}236 \pm 43 \\
(132-319)\end{array}$ & $\begin{array}{c}5.9 \pm 1.4 \\
(3-9)\end{array}$ & $\begin{array}{c}498 \pm 126 \\
(195-819)\end{array}$ & .36 & .36 & $.19-.51$ & .13 & $<.001$ \\
\hline
\end{tabular}

Table 2. Mean and SD (and range) of Edwards, RPE, and session-RPE parameters (Arbitrary Units, AU), and corresponding correlations between session-RPE and Edward's values (ICC, $r, 95 \%$ C.I., $R^{2}$, and $p$ values) of each type of training session in terms of gender and competition level (i.e., elite or amateur), gender and type of main workout (i.e., conditioning, technical, tactical/ game), and competition level (i.e., elite or amateur) and type of session (i.e., conditioning, technical, tactical/game)

\begin{tabular}{|c|c|c|c|c|c|c|c|c|c|c|}
\hline \multirow{2}{*}{$\begin{array}{l}\text { Gender } \\
\text { subgroup }\end{array}$} & \multirow{2}{*}{$\begin{array}{c}\text { Competition } \\
\text { level } \\
\text { subgroup }\end{array}$} & \multirow{2}{*}{$\begin{array}{l}\text { Type of } \\
\text { session } \\
\text { subgroup }\end{array}$} & \multirow{2}{*}{$\begin{array}{l}\text { Edwards } \\
\text { (AU) }\end{array}$} & \multirow{2}{*}{$\begin{array}{l}\text { RPE } \\
\text { (AU) }\end{array}$} & \multirow{2}{*}{$\begin{array}{l}\text { Session-RPE } \\
\text { (AU) }\end{array}$} & \multicolumn{5}{|c|}{$\begin{array}{l}\text { Edwards - session-RPE } \\
\text { correlation indexes }\end{array}$} \\
\hline & & & & & & ICC & $r$ & 95\% C.I. & $R^{2}$ & $p$ \\
\hline Female & Elite & $\begin{array}{c}\text { All sessions } \\
\quad(n=93)\end{array}$ & $\begin{array}{c}216 \pm 59 \\
(61-300)\end{array}$ & $\begin{array}{l}6 \pm 1.7 \\
(3-10)\end{array}$ & $\begin{array}{l}442 \pm 124 \\
(204-710)\end{array}$ & .75 & .77 & $.67-.84$ & .6 & $<.001$ \\
\hline Female & Amateur & $\begin{array}{l}\text { All sessions } \\
\quad(n=94)\end{array}$ & $\begin{array}{c}203 \pm 57 \\
(108-324)\end{array}$ & $\begin{array}{c}5.9 \pm 1.5 \\
(3-10)\end{array}$ & $\begin{array}{l}439 \pm 134 \\
(180-720)\end{array}$ & .7 & .75 & $.64-.83$ & .56 & $<.001$ \\
\hline Male & Elite & $\begin{array}{l}\text { All sessions } \\
\quad(n=104)\end{array}$ & $\begin{array}{l}204 \pm 62 \\
(67-296)\end{array}$ & $\begin{array}{l}5.5 \pm 1.5 \\
(2-8)\end{array}$ & $\begin{array}{c}413 \pm 131 \\
(134-736)\end{array}$ & .75 & .77 & $.68-.84$ & .59 & $<.001$ \\
\hline Male & Amateur & $\begin{array}{c}\text { All sessions } \\
(n=100)\end{array}$ & $\begin{array}{c}218 \pm 60 \\
(120-360) \\
\end{array}$ & $\begin{array}{c}6.4 \pm 1.5 \\
(3-9) \\
\end{array}$ & $\begin{array}{l}478 \pm 150 \\
(180-819) \\
\end{array}$ & .68 & .74 & $.64-.82$ & .55 & $<.001$ \\
\hline Female & All sessions & $\begin{array}{l}\text { Conditioning } \\
(\mathrm{n}=65)\end{array}$ & $\begin{array}{c}248 \pm 44 \\
(108-324)\end{array}$ & $\begin{array}{c}7.4 \pm 1.3 \\
(5-10)\end{array}$ & $\begin{array}{c}532 \pm 87 \\
(358-720)\end{array}$ & .7 & .67 & $.51-.79$ & .45 & $<.001$ \\
\hline Female & All sessions & $\begin{array}{l}\text { Technical } \\
(\mathrm{n}=70)\end{array}$ & $\begin{array}{c}160 \pm 48 \\
(61-285)\end{array}$ & $\begin{array}{l}4.7 \pm 1 \\
(3-7)\end{array}$ & $\begin{array}{l}335 \pm 109 \\
(180-665)\end{array}$ & .67 & .68 & $.53-.79$ & .46 & $<.001$ \\
\hline Female & All sessions & $\begin{array}{l}\text { Tactical/game } \\
\quad(n=52)\end{array}$ & $\begin{array}{c}227 \pm 35 \\
(137-308)\end{array}$ & $\begin{array}{l}5.7 \pm 1 \\
(3-8)\end{array}$ & $\begin{array}{c}466 \pm 92 \\
(273-704)\end{array}$ & .22 & .19 & $-.09-.44$ & .03 & $>.05$ \\
\hline Male & All sessions & $\begin{array}{l}\text { Conditioning } \\
(\mathrm{n}=69)\end{array}$ & $\begin{array}{c}242 \pm 41 \\
(150-360)\end{array}$ & $\begin{array}{c}6.8 \pm 1.2 \\
(4-9)\end{array}$ & $\begin{array}{l}499 \pm 90 \\
(292-704)\end{array}$ & .77 & .82 & $.72-.88$ & .68 & $<.001$ \\
\hline Male & All sessions & $\begin{array}{l}\text { Technical } \\
(\mathrm{n}=67)\end{array}$ & $\begin{array}{c}146 \pm 37 \\
(67-225)\end{array}$ & $\begin{array}{c}4.8 \pm 1 \\
(2-7)\end{array}$ & $\begin{array}{c}308 \pm 78 \\
(134-480)\end{array}$ & .58 & .53 & $.33-.68$ & .28 & $<.001$ \\
\hline Male & All sessions & $\begin{array}{c}\text { Tactical/game } \\
(\mathrm{n}=68)\end{array}$ & $\begin{array}{c}243 \pm 48 \\
(132-319)\end{array}$ & $\begin{array}{c}6.1 \pm 1.6 \\
(3-9)\end{array}$ & $\begin{array}{c}523 \pm 143 \\
(195-819) \\
\end{array}$ & .37 & .38 & $.16-.57$ & .14 & .001 \\
\hline All sessions & Elite & $\begin{array}{l}\text { Conditioning } \\
\quad(n=69)\end{array}$ & $\begin{array}{c}241 \pm 32 \\
(179-300)\end{array}$ & $\begin{array}{c}6.9 \pm 1.5 \\
(4-10)\end{array}$ & $\begin{array}{c}489 \pm 93 \\
(292-710)\end{array}$ & .69 & .85 & $.77-.9$ & .73 & $<.001$ \\
\hline All sessions & Elite & $\begin{array}{l}\text { Technical } \\
(\mathrm{n}=70)\end{array}$ & $\begin{array}{l}150 \pm 54 \\
(61-285)\end{array}$ & $\begin{array}{c}4.5 \pm 1.2 \\
(2-7)\end{array}$ & $\begin{array}{c}338 \pm 118 \\
(134-665)\end{array}$ & .69 & .69 & $.54-.8$ & .48 & $<.001$ \\
\hline All sessions & Elite & $\begin{array}{l}\text { Tactical/game } \\
(\mathrm{n}=58)\end{array}$ & $\begin{array}{c}244 \pm 33 \\
(188-296)\end{array}$ & $\begin{array}{c}5.8 \pm 1.3 \\
(3-8)\end{array}$ & $\begin{array}{c}458 \pm 117 \\
(195-736)\end{array}$ & .48 & .59 & $.39-.74$ & .35 & $<.001$ \\
\hline All sessions & Amateur & $\begin{array}{l}\text { Conditioning } \\
\quad(n=65)\end{array}$ & $\begin{array}{c}249 \pm 51 \\
(108-360)\end{array}$ & $\begin{array}{c}7.4 \pm 1.0 \\
(5-10)\end{array}$ & $\begin{array}{c}543 \pm 77 \\
(360-720)\end{array}$ & .82 & .76 & $.63-.85$ & .57 & $<.001$ \\
\hline All sessions & Amateur & $\begin{array}{c}\text { Technical } \\
(\mathrm{n}=62)\end{array}$ & $\begin{array}{c}157 \pm 28 \\
(117-228)\end{array}$ & $\begin{array}{l}5 \pm 0.9 \\
(3-7)\end{array}$ & $\begin{array}{c}306 \pm 61 \\
(180-455)\end{array}$ & .58 & .54 & $.33-.7$ & .29 & $<.001$ \\
\hline All sessions & Amateur & $\begin{array}{l}\text { Tactical/game } \\
(\mathrm{n}=67)\end{array}$ & $\begin{array}{c}228 \pm 50 \\
(132-319)\end{array}$ & $\begin{array}{c}6.1 \pm 1.4 \\
(3-9)\end{array}$ & $\begin{array}{c}536 \pm 124 \\
(264-819)\end{array}$ & .41 & .37 & $.14-.56$ & .13 & .003 \\
\hline
\end{tabular}


Strong differences emerged also between the observed types of sessions $(\mathrm{p}<.001$, ES range $=0.7)$, highlighting lower Edwards' ITLs of the technical sessions with respect to the conditioning $(\mathrm{p}<.001$, $\mathrm{ES}=0.7)$ and tactical/game $(\mathrm{p}<.001, \mathrm{ES}=.7)$ ones (Table 1). The same trend determined the significance regarding the interactions of type of session with gender $(p=.043$, ES range $=0.6-0.8)$, and competition level $(\mathrm{p}=.007, \mathrm{ES}=0.7)$. In particular, Edwards' ITLs resulted strongly lower in the technical sessions than in the conditioning and tactical/game ones associated to both the female $(\mathrm{p}<.001, \mathrm{ES}$ range $=0.6-0.7)$ and male $(\mathrm{p}<.001, \mathrm{ES}$ range $=0.7-0.8)$, and amateur $(\mathrm{p}<.001, \mathrm{ES}=0.7)$ and elite $(\mathrm{p}<.001$, ES $=0.7)$ competition level subgroups (Table 2). Finally, no difference for Edwards' ITLs emerged in the interaction of all the three observed variables (Table 3).

For the correlation between the ITLs assessed by the session-RPE and Edwards' methods, very large relationships emerged for both genders, whereas for the competition level, very large and large relationships were reported for elite and amateur subgroups, respectively; finally, in terms of the type of sessions, very large, large, and moderate correlations were found for the conditioning, technical, and tactical/ game sessions, respectively (Table 1).
For the sessions related to the interaction of two variables (over the three observed), the grade of correlation between the two methods resulted in very large or large correlations, with the exception of the tactical/game sessions performed by the male, elite, and amateur players subgroups, which resulted only in moderate correlations, and of the tactical/game sessions performed by all females, which even resulted in not significant correlations (Table 2). In addition, very large and large relationships between the two observed methods were also reported for almost all the session subgroups related to the interaction of all the three variables. The exceptions emerged for the tactical/game sessions performed by the amateur male and female beach volleyball players, which were moderate and not significant, respectively (Table 3 ).

The analysis applied for each single player (Table 4) reported very large and large correlations between the ITLs related to the two observed methods for almost every case (with four almost perfect individual correlations). However, five individual cases were not significant. SessionRPE and Edwards' values regarding each elite and amateur training session (regardless of gender) were portrayed in Figures 1a and 1b, respectively.

Table 3. Mean and SD (and range) of Edwards, RPE, and session-RPE parameters (Arbitrary Units, AU), and corresponding correlations between session-RPE and Edward's values (ICC, $r, 95 \%$ C.I., $R^{2}$, and $p$ values) of each type of training session in terms of gender, competition level (i.e., elite or amateur), and type of session (i.e., conditioning, technical, tactical/game)

\begin{tabular}{|c|c|c|c|c|c|c|c|c|c|c|}
\hline \multirow{2}{*}{$\begin{array}{l}\text { Gender } \\
\text { subgroup }\end{array}$} & \multirow{2}{*}{$\begin{array}{c}\text { Competition } \\
\text { level } \\
\text { subgroup }\end{array}$} & \multirow{2}{*}{$\begin{array}{l}\text { Type of } \\
\text { session } \\
\text { subgroup }\end{array}$} & \multirow{2}{*}{$\begin{array}{l}\text { Edwards } \\
\text { (AU) }\end{array}$} & \multirow{2}{*}{$\begin{array}{l}\text { RPE } \\
\text { (AU) }\end{array}$} & \multirow{2}{*}{$\begin{array}{l}\text { Session-RPE } \\
\text { (AU) }\end{array}$} & \multicolumn{5}{|c|}{$\begin{array}{l}\text { Edwards - session-RPE } \\
\text { correlation indexes }\end{array}$} \\
\hline & & & & & & ICC & $r$ & 95\% C.I. & $R^{2}$ & $p$ \\
\hline Female & Elite & $\begin{array}{l}\text { Conditioning } \\
\quad(n=34)\end{array}$ & $\begin{array}{c}254 \pm 31 \\
(179-300)\end{array}$ & $\begin{array}{l}7.4 \pm 1.5 \\
(5-10)\end{array}$ & $\begin{array}{c}521 \pm 95 \\
(358-710)\end{array}$ & .65 & .8 & $.63-.9$ & .64 & $<.001$ \\
\hline Female & Elite & $\begin{array}{l}\text { Technical } \\
(n=36)\end{array}$ & $\begin{array}{c}166 \pm 61 \\
(61-285)\end{array}$ & $\begin{array}{c}4.6 \pm 1.1 \\
(3-7)\end{array}$ & $\begin{array}{c}371 \pm 132 \\
(204-665)\end{array}$ & .7 & .71 & $.5-.84$ & .5 & $<.001$ \\
\hline Female & Elite & $\begin{array}{l}\text { Tactical/game } \\
\quad(n=23)\end{array}$ & $\begin{array}{c}239 \pm 20 \\
(204-276)\end{array}$ & $\begin{array}{c}5.9 \pm 0.8 \\
(4-7)\end{array}$ & $\begin{array}{c}435 \pm 72 \\
(345-634)\end{array}$ & .46 & .58 & $.22-.8$ & .33 & .004 \\
\hline Female & Amateur & $\begin{array}{l}\text { Conditioning } \\
(\mathrm{n}=31)\end{array}$ & $\begin{array}{c}242 \pm 54 \\
(108-324)\end{array}$ & $\begin{array}{c}7.5 \pm 1.0 \\
(6-10)\end{array}$ & $\begin{array}{c}544 \pm 78 \\
(432-720)\end{array}$ & .8 & .71 & $.47-.85$ & .5 & $<.001$ \\
\hline Female & Amateur & $\begin{array}{l}\text { Technical } \\
(\mathrm{n}=34)\end{array}$ & $\begin{array}{c}154 \pm 30 \\
(117-228)\end{array}$ & $\begin{array}{c}4.9 \pm 0.9 \\
(3-7)\end{array}$ & $\begin{array}{c}298 \pm 59 \\
(180-420)\end{array}$ & .61 & .54 & $.25-.74$ & .29 & .001 \\
\hline Female & Amateur & $\begin{array}{l}\text { Tactical/game } \\
(n=29)\end{array}$ & $\begin{array}{c}217 \pm 41 \\
(137-308)\end{array}$ & $\begin{array}{c}5.6 \pm 1.1 \\
(3-8)\end{array}$ & $\begin{array}{l}491 \pm 100 \\
(273-704)\end{array}$ & .29 & .24 & $-.14-.56$ & .06 & $>.05$ \\
\hline Male & Elite & $\begin{array}{l}\text { Conditioning } \\
(\mathrm{n}=35)\end{array}$ & $\begin{array}{c}229 \pm 27 \\
(180-277)\end{array}$ & $\begin{array}{c}6.3 \pm 1.1 \\
(4-8)\end{array}$ & $\begin{array}{c}458 \pm 82 \\
(292-584)\end{array}$ & .69 & .88 & $.77-.94$ & .77 & $<.001$ \\
\hline Male & Elite & $\begin{array}{l}\text { Technical } \\
(n=34)\end{array}$ & $\begin{array}{c}133 \pm 41 \\
(67-221)\end{array}$ & $\begin{array}{c}4.5 \pm 1.2 \\
(2-6)\end{array}$ & $\begin{array}{c}302 \pm 91 \\
(134-480)\end{array}$ & .58 & .55 & $.26-.75$ & .3 & .001 \\
\hline Male & Elite & $\begin{array}{l}\text { Tactical/game } \\
\quad(n=35)\end{array}$ & $\begin{array}{c}248 \pm 39 \\
(188-296)\end{array}$ & $\begin{array}{c}5.7 \pm 1.6 \\
(3-8)\end{array}$ & $\begin{array}{c}474 \pm 138 \\
(195-736)\end{array}$ & .47 & .59 & $.57-.87$ & .34 & $<.001$ \\
\hline Male & Amateur & $\begin{array}{l}\text { Conditioning } \\
\quad(n=34)\end{array}$ & $\begin{array}{c}255 \pm 48 \\
(150-360)\end{array}$ & $\begin{array}{c}7.4 \pm 1.0 \\
(5-9)\end{array}$ & $\begin{array}{c}542 \pm 78 \\
(360-704)\end{array}$ & .85 & .82 & $.67-.91$ & .68 & $<.001$ \\
\hline Male & Amateur & $\begin{array}{c}\text { Technical } \\
(n=33)\end{array}$ & $\begin{array}{c}160 \pm 26 \\
(120-225)\end{array}$ & $\begin{array}{c}5.1 \pm 1.0 \\
(3-7)\end{array}$ & $\begin{array}{c}314 \pm 62 \\
(180-455)\end{array}$ & .55 & .53 & $.23-.74$ & .28 & .001 \\
\hline Male & Amateur & $\begin{array}{l}\text { Tactical/game } \\
(n=33)\end{array}$ & $\begin{array}{c}237 \pm 55 \\
(132-319)\end{array}$ & $\begin{array}{c}6.5 \pm 1.5 \\
(3-9)\end{array}$ & $\begin{array}{c}575 \pm 131 \\
(264-819)\end{array}$ & .42 & .37 & $.03-.67$ & .13 & .036 \\
\hline
\end{tabular}



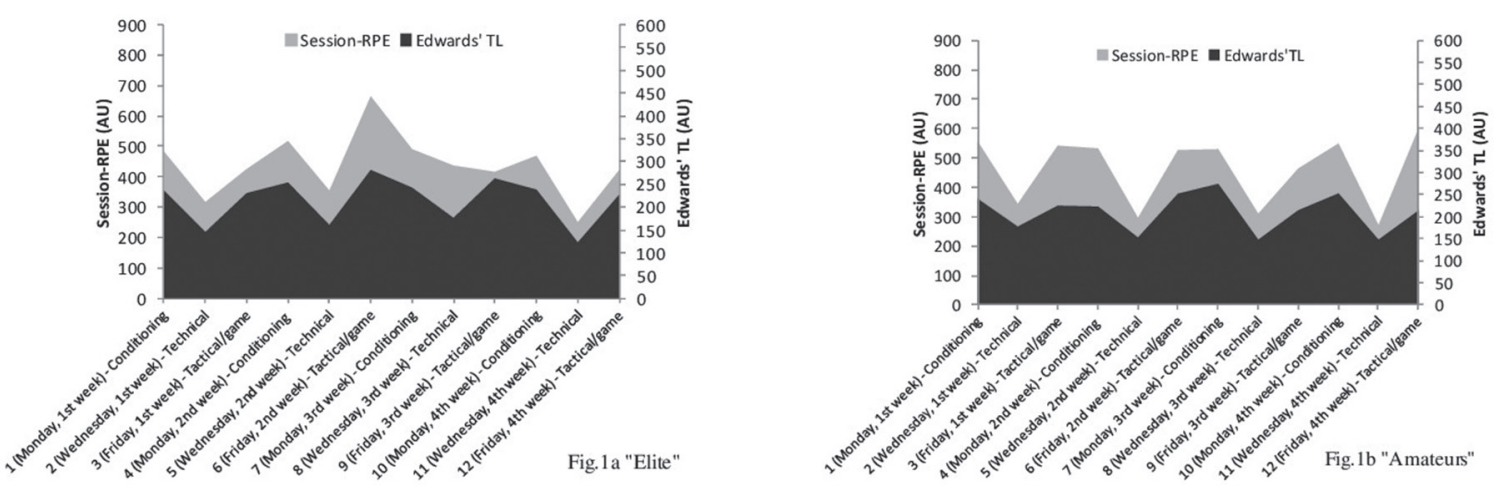

Figure $1(a, b)$. Session rating of perceived exertion (RPE) and Edwards' training load (TL) for elite (Figure la) and amateur (Figure 1b) players during 12 training sessions. Abbreviation AU indicates arbitrary unit.

Table 4. Mean and SD (and range) of Edwards, RPE, and session-RPE parameters (Arbitrary Units, AU), and corresponding correlations between session-RPE and Edward's values (ICC, $r, 95 \%$ C.I., $R^{2}$, and $p$ values) for each beach volleyball player

\begin{tabular}{|c|c|c|c|c|c|c|c|c|c|}
\hline \multirow{2}{*}{ Subgroup } & \multirow{2}{*}{$\begin{array}{c}\# \\
\text { Player } \\
\text { (n sessions) }\end{array}$} & \multirow{2}{*}{$\begin{array}{l}\text { Edwards } \\
\text { (AU) }\end{array}$} & \multirow{2}{*}{$\begin{array}{l}\text { RPE } \\
(\mathrm{AU})\end{array}$} & \multirow{2}{*}{$\begin{array}{l}\text { Session-RPE } \\
\text { (AU) }\end{array}$} & \multicolumn{5}{|c|}{$\begin{array}{l}\text { Edwards - session-RPE } \\
\text { correlation indexes }\end{array}$} \\
\hline & & & & & ICC & $r$ & 95\% C.I. & $R^{2}$ & $p$ \\
\hline \multirow{9}{*}{$\begin{array}{l}\text { Female, } \\
\text { Elite }\end{array}$} & $\# 1$ (10) & $\begin{array}{c}224 \pm 57 \\
(121-285)\end{array}$ & $6 \pm 1(4-8)$ & $\begin{array}{l}453 \pm 118 \\
(272-634)\end{array}$ & .82 & .88 & $.56-.97$ & .78 & $.001^{*}$ \\
\hline & \#2 (11) & $\begin{array}{c}221 \pm 51 \\
(102-273)\end{array}$ & $6 \pm 2(3-10)$ & $\begin{array}{l}453 \pm 140 \\
(204-710)\end{array}$ & .63 & .71 & $.19-.92$ & .51 & $.014^{*}$ \\
\hline & \#3 (11) & $\begin{array}{l}202 \pm 65 \\
(91-278)\end{array}$ & $6 \pm 2(3-10)$ & $\begin{array}{c}428 \pm 137 \\
(204-665)\end{array}$ & .71 & .76 & $.30-.93$ & .58 & $.007^{*}$ \\
\hline & \#4 (10) & $\begin{array}{l}219 \pm 74 \\
(61-300)\end{array}$ & $7 \pm 2(4-10)$ & $\begin{array}{l}477 \pm 137 \\
(272-650\end{array}$ & .82 & .83 & $.42-.96$ & .69 & $.003^{*}$ \\
\hline & \#5 (11) & $\begin{array}{c}222 \pm 65 \\
(102-285)\end{array}$ & $6 \pm 2(4-9)$ & $\begin{array}{l}441 \pm 108 \\
(272-639)\end{array}$ & .87 & .87 & $.57-.97$ & .76 & $<.001^{*}$ \\
\hline & \#6 (10) & $\begin{array}{c}225 \pm 57 \\
(121-289)\end{array}$ & $6 \pm 2(4-9)$ & $\begin{array}{c}427 \pm 122 \\
(242-639)\end{array}$ & .76 & .80 & $.34-.95$ & .64 & $.006^{*}$ \\
\hline & \#7 (11) & $\begin{array}{c}206 \pm 61 \\
(102-285)\end{array}$ & $6 \pm 2(4-9)$ & $\begin{array}{c}428 \pm 94 \\
(272-585)\end{array}$ & .77 & .68 & $.14-.91$ & .47 & $.020^{*}$ \\
\hline & \#8 (9) & $\begin{array}{c}208 \pm 54 \\
(121-274)\end{array}$ & $6 \pm 2(3-9)$ & $\begin{array}{l}449 \pm 138 \\
(204-665)\end{array}$ & .59 & .62 & $-.08-.91$ & .39 & .075 \\
\hline & \#9 (10) & $\begin{array}{c}217 \pm 67 \\
(102-293) \\
\end{array}$ & $6 \pm 2(3-10)$ & $\begin{array}{c}419 \pm 139 \\
(204-650) \\
\end{array}$ & .84 & .93 & $.73-.98$ & .87 & $<.001^{*}$ \\
\hline \multirow{9}{*}{$\begin{array}{l}\text { Male, } \\
\text { Elite }\end{array}$} & \#1 (11) & $\begin{array}{l}210 \pm 53 \\
122-285\end{array}$ & $6 \pm 2(3-8)$ & $\begin{array}{l}413 \pm 146 \\
(201-644)\end{array}$ & .69 & .81 & $.41-.95$ & .66 & $.003^{*}$ \\
\hline & \#2 (12) & $\begin{array}{l}203 \pm 64 \\
(67-292)\end{array}$ & $5 \pm 2(2-7)$ & $\begin{array}{l}360 \pm 124 \\
(134-552)\end{array}$ & .81 & .83 & $.49-.95$ & .69 & $.001^{*}$ \\
\hline & \#3 (12) & $\begin{array}{l}208 \pm 66 \\
(92-296)\end{array}$ & $6 \pm 2(3-6)$ & $\begin{array}{c}420 \pm 145 \\
(183-736)\end{array}$ & .76 & .81 & $.44-.94$ & .66 & $.001^{*}$ \\
\hline & \#4 (11) & $\begin{array}{l}206 \pm 71 \\
(67-295)\end{array}$ & $5 \pm 1(3-7)$ & $\begin{array}{l}402 \pm 123 \\
(201-552)\end{array}$ & .88 & .91 & $.68-.98$ & .83 & $<.001^{*}$ \\
\hline & \#5 (12) & $\begin{array}{l}209 \pm 61 \\
(92-293)\end{array}$ & $6 \pm 1(3-8)$ & $\begin{array}{l}435 \pm 117 \\
(244-644)\end{array}$ & .78 & .77 & $.35-.93$ & .60 & $.003^{*}$ \\
\hline & \#6 (12) & $\begin{array}{c}195 \pm 74 \\
(67-292)\end{array}$ & $6 \pm 2(3-8)$ & $\begin{array}{l}428 \pm 148 \\
(201-736)\end{array}$ & .77 & .78 & $.37-.94$ & .61 & $.003^{*}$ \\
\hline & \#7 (11) & $\begin{array}{c}208 \pm 61 \\
(101-292)\end{array}$ & $6 \pm 1(4-8)$ & $\begin{array}{l}441 \pm 125 \\
(268-736)\end{array}$ & .74 & .74 & $.25-.93$ & .55 & $.009^{*}$ \\
\hline & \#8 (12) & $\begin{array}{c}193 \pm 64 \\
(67-289)\end{array}$ & $5 \pm 2(3-7)$ & $\begin{array}{c}377 \pm 137 \\
(195-644)\end{array}$ & .69 & .69 & $.19-.91$ & .47 & $.014^{*}$ \\
\hline & \#9 (11) & $\begin{array}{c}205 \pm 60 \\
(122-288)\end{array}$ & $6 \pm 1(4-8)$ & $\begin{array}{c}441 \pm 130 \\
(244-736)\end{array}$ & .72 & .74 & $.25-.93$ & .55 & $.009^{*}$ \\
\hline
\end{tabular}


Continuation of Table 4

\begin{tabular}{|c|c|c|c|c|c|c|c|c|c|}
\hline \multirow{2}{*}{ Subgroup } & \multirow{2}{*}{$\begin{array}{c}\# \\
\text { Player } \\
\text { (n sessions) }\end{array}$} & \multirow{2}{*}{$\begin{array}{l}\text { Edwards } \\
\text { (AU) }\end{array}$} & \multirow{2}{*}{$\begin{array}{l}\text { RPE } \\
\text { (AU) }\end{array}$} & \multirow{2}{*}{$\begin{array}{l}\text { Session-RPE } \\
\text { (AU) }\end{array}$} & \multicolumn{5}{|c|}{$\begin{array}{l}\text { Edwards - session-RPE } \\
\text { correlation indexes }\end{array}$} \\
\hline & & & & & ICC & $r$ & 95\% C.I. & $R^{2}$ & $p$ \\
\hline \multirow{9}{*}{$\begin{array}{l}\text { Female, } \\
\text { Amateur }\end{array}$} & \#1 (9) & $\begin{array}{c}181 \pm 49 \\
(120-259)\end{array}$ & $6 \pm 1(4-8)$ & $\begin{array}{l}455 \pm 155 \\
(240-637)\end{array}$ & .71 & .93 & $.70-.99$ & .87 & $<.001^{*}$ \\
\hline & \#2 (11) & $\begin{array}{c}211 \pm 57 \\
(137-307)\end{array}$ & $6 \pm 2(4-5)$ & $\begin{array}{l}468 \pm 146 \\
(240-648)\end{array}$ & .52 & .51 & $.13-.85$ & .26 & .107 \\
\hline & \#3 (11) & $\begin{array}{c}223 \pm 65 \\
(146-324)\end{array}$ & $6 \pm 2(4-10)$ & $\begin{array}{c}472 \pm 137(240- \\
720)\end{array}$ & .80 & .85 & $.51-.96$ & .73 & $.001^{*}$ \\
\hline & $\# 4$ (8) & $\begin{array}{c}183 \pm 50 \\
(137-288)\end{array}$ & $6 \pm 1(4-8)$ & $\begin{array}{l}429 \pm 138 \\
(240-584)\end{array}$ & .61 & .68 & $-.05-.94$ & .46 & .064 \\
\hline & \#5 (10) & $\begin{array}{c}208 \pm 57 \\
(120-292)\end{array}$ & $6 \pm 1(4-9)$ & $\begin{array}{c}461 \pm 128 \\
(240-657)\end{array}$ & .82 & .92 & $.69-.98$ & .85 & $<.001^{*}$ \\
\hline & \#6 (9) & $\begin{array}{c}185 \pm 50 \\
(108-256)\end{array}$ & $6 \pm 1(4-8)$ & $\begin{array}{c}410 \pm 96 \\
(240-584)\end{array}$ & .65 & .59 & $-.12-.90$ & .35 & .094 \\
\hline & \#7 (11) & $\begin{array}{c}192 \pm 52 \\
(148-300)\end{array}$ & $5 \pm 2(3-8)$ & $\begin{array}{l}385 \pm 136 \\
(180-600)\end{array}$ & .64 & .70 & $.13-.92$ & .49 & $.016^{*}$ \\
\hline & \#8 (9) & $\begin{array}{c}190 \pm 59 \\
(120-288)\end{array}$ & $6 \pm 2(3-8)$ & $\begin{array}{c}398 \pm 131 \\
(180-600)\end{array}$ & .78 & .84 & $.40-.97$ & .71 & $.004^{*}$ \\
\hline & \#9 (11) & $\begin{array}{c}211 \pm 67 \\
(117-300) \\
\end{array}$ & $6 \pm 2(3-9)$ & $\begin{array}{l}434 \pm 150 \\
(180-675) \\
\end{array}$ & .77 & .83 & $.46-.95$ & .69 & $.002 *$ \\
\hline \multirow{9}{*}{$\begin{array}{l}\text { Male, } \\
\text { Amateur }\end{array}$} & \#1 (11) & $\begin{array}{c}217 \pm 49 \\
(144-308)\end{array}$ & $7 \pm 1(5-8)$ & $\begin{array}{c}514 \pm 154(300- \\
728)\end{array}$ & .35 & .37 & $-.30-.79$ & .14 & .263 \\
\hline & \#2 (11) & $\begin{array}{c}232 \pm 67 \\
(120-300)\end{array}$ & $7 \pm 2(4-9)$ & $\begin{array}{c}511 \pm 179 \\
(300-819)\end{array}$ & .72 & .87 & $.57-.97$ & .75 & $.001^{*}$ \\
\hline & \#3 (12) & $\begin{array}{c}228 \pm 57 \\
(130-319)\end{array}$ & $6 \pm 2(3-9)$ & $\begin{array}{l}459 \pm 179 \\
(240-819)\end{array}$ & .61 & .76 & $.33-.93$ & .57 & $.004^{*}$ \\
\hline & $\# 4$ (10) & $\begin{array}{c}209 \pm 68 \\
(147-307)\end{array}$ & $6 \pm 2(4-9)$ & $\begin{array}{l}469 \pm 159 \\
(240-765)\end{array}$ & .76 & .84 & $.45-.96$ & .70 & $.002^{*}$ \\
\hline & \#5 (12) & $\begin{array}{c}218 \pm 62 \\
(148-324)\end{array}$ & $6 \pm 2(4-9)$ & $\begin{array}{l}470 \pm 157 \\
(240-728)\end{array}$ & .65 & .71 & $.23-.91$ & .50 & $.010^{*}$ \\
\hline & \#6 (12) & $\begin{array}{c}233 \pm 75 \\
(120-360)\end{array}$ & $7 \pm 1(4-9)$ & $\begin{array}{l}503 \pm 154 \\
(240-728)\end{array}$ & .82 & .88 & $.62-.97$ & .78 & $<.001^{*}$ \\
\hline & \#7 (11) & $\begin{array}{c}206 \pm 68 \\
(120-324)\end{array}$ & $6 \pm 2(3-9)$ & $\begin{array}{l}468 \pm 146 \\
(180-657)\end{array}$ & .73 & .75 & $.27-.93$ & .56 & $.008^{*}$ \\
\hline & \#8 (10) & $\begin{array}{c}211 \pm 53 \\
(120-288)\end{array}$ & $6 \pm 1(4-8)$ & $\begin{array}{c}467 \pm 114 \\
(240-595)\end{array}$ & .66 & .65 & $.04-.91$ & .42 & $.042^{*}$ \\
\hline & \#9 (11) & $\begin{array}{c}201 \pm 52 \\
(120-307)\end{array}$ & $6 \pm 1(3-7)$ & $\begin{array}{c}436 \pm 125 \\
(180-595)\end{array}$ & .72 & .79 & $.36-.94$ & .63 & $.004^{*}$ \\
\hline
\end{tabular}

\section{Discussion and conclusions}

To our knowledge, this is the first study focused on the correlation between session-RPE and Edwards' method for quantifying ITLs in beach volleyball players. The main finding of this study was that session-RPE is a valuable tool to monitor beach volleyball players regardless of gender, competition level, and type of session performed, highlighting a strong relationship with the criterion of measurement (i.e., Edwards' method), in line with what has been reported in other team and situational sports such as basketball (Lupo, et al., 2017b), water polo (Lupo, et al., 2014), soccer (Impellizzeri, et al., 2004), and Australian football (Scott, Black, Quinn, and Coutts, 2013). However, some limitations seem to affect the tactical/game sessions, for which only moderate correlations between sessionRPE and Edwards' method emerged.

A very large correlation between session-RPE and Edwards' method has been reported both in male and female sessions, probably because their ITLs were quite similar. Coherently to gender, the same picture has been shown for the competition level subcategories, making session-RPE a valuable tool to monitor training loads regardless of beach volleyball players' experience. Despite no gender and competition level effect emerged for Edwards' ITLs, their subgroup interactions were different (with moderate ESs), reporting the lowest values for the technical sessions. Therefore, this result underpins the assertion that the type of session can substantially influence the ITL, even 
more than gender and competition level. However, further evidences are needed to confirm or contrast this effect for other sport and training scenarios.

Although the Edwards' ITLs of the technical sessions were considerably lower (with moderate ESs) than the other session types, its corresponding correlation between session-RPE and Edwards' method was highly satisfactory. Differently, for the tactical/game training sessions, session-RPE was moderately correlated with the Edwards' method, although ITLs were similar to that of the conditioning sessions. As a consequence, the two observed methods are correlated regardless of the ITL level.

However, the session-RPE validity values reported in this study for the three types of sessions result coherent to a previous study (Lupo, et al., 2014), where the tactical/game sessions were recognized as the portion of training in which players probably experienced what they really preferred to perform during a training session, feeling pleasure from practice, and therefore, probably underestimating their RPE scores. Nevertheless, a different scenario for a similar analysis emerged in youth basketball (Lupo, et al., 2017b), where players reported strong relationships between the two methods in every session type. Therefore, further studies should clarify if the session-RPE validity is really limited for the tactical/game sessions. Workrest analysis of training sessions and a training dairy, especially focused on discriminating technical from tactical sessions, characterized by frequent or rare coaches' explanatory interruptions, could contribute to better understanding of this issue.

Weak relationships between sessions-RPE and Edwards' values related to the tactical/game sessions emerged especially when associated with the female and female-amateur subgroups. In addition, this effect seems to be further confirmed by the analysis on individual players' sessions (Table 4) where significant correlations between the two observed methods emerged more frequently in the male (17 over 18 cases) than in the female (14 over 18 cases) subgroup, and more in the elite (17 over 18 cases) than in the amateur (14 over 18 cases) players. Therefore, even though the general correlation between session-RPE and Edwards' method related to female and amateur player subgroups gave satisfactory results, individual players appertaining to these categories seemed only partially able to evaluate their own RPE.

The main limitation of this study was the absence of the individual HRmax directly evaluated by incremental all-out tests, thus this parameter was estimated by means of an often-criticized formula. In fact, "220-age" formula has been considered as too generic to quantify the true level of physical stress (Tanaka, Monahan, \& Seals, 2001), which should be inferred according to a sport-specific equation (Nikolaidis, 2015).

From a practical point of view, the use of the session-RPE method in beach volleyball players' ITL assessment can improve the quality of training sessions. In fact, in case of low ITL, coaches will be able to provide higher and adequate stimuli for effectively improving player's performance level; conversely, for excessive RPE scores, coaches will be able to reduce training loads, thus avoiding the risks of overreaching and injuries. Similarly, a high utility of the session-RPE method can be considered also in amateur training, where players' performances are less known to coaches and probably characterized by a high heterogeneity, due to different training backgrounds, recovery capability, and lifestyles.

In conclusion, this study has demonstrated that the session-RPE method is a valuable method for monitoring ITL in beach volleyball players of both genders as well as of both elite and amateur standard. On the other hand, session-RPE seems to be a valuable method to monitor beach volleyball players' ITL only for the conditioning and technical sessions, reporting limitations for the tactical/game sessions, which should be better clarify in further studies.

\section{References}

Aoki, M.S., Arruda, A.F.S., Freitas, C.G., Miloski, B., Marcelino, P.R., Drago, G., Drago, M., \& Moreira, A. (2017). Monitoring training loads, mood states, and jump performance over two periodized training mesocycles in elite young volleyball players. International Journal of Sports Science and Coaching, 12, 130-137. doi: $10.1177 / 1747954116684394$

Bangsbo, J. (2000). Team sports. In R. Maughan (Ed.), Nutrition in sports. Oxford: Blackwell Science.

Borg, G. (1998). Borg's perceived exertion and pain scales. Champaign, IL: Human Kinetics.

Casolino, E., Cortis, C., Lupo, C., Chiodo, S., Minganti, C., \& Capranica, L. (2012). Physiological versus psychological evaluation in taekwondo elite athletes. International Journal of Sports Physiology and Performance, 7(4), 322-331. doi: 10.1123/ijspp.7.4.322

Cohen, J. (1988). Statistical power analysis for the behavioral sciences (2 $2^{\text {nd }}$ ed.). Hillsdale, NJ: Lawrence Erlbaum Associates. 
Coutts, A.J., Rampinini, E., Marcora, S.M., Castagna, C., \& Impellizzeri, F.M. (2009). Heart rate and blood lactate correlates of perceived exertion during small-sided soccer games. Journal of Science and Medicine in Sport, 12(1), 79-84. doi: 10.1016/j.jsams.2007.08.005

Coutts, A.J., Vitasovic Gomes, R., Viveiros, L., \& Aoki, M.S. (2010). Monitoring training loads in elite tennis. Revista Brasileira de Cineantropometria \& Desempenho Humano, 12(3), 217-220. doi: 10.5007/1980-0037.2010v12n3p217

Edwards, S. (1993). The heart rate monitor book. Sacramento, CA: Fleet Feet.

Foster, C. (1998). Monitoring training in athletes with reference to overtraining syndrome. Medicine and Science in Sports and Exercise, 30, 1164-1168. doi: 10.1097/00005768-199807000-00023

Foster, C., Hector, L., Welsh, R., Schrager, M., Green, M., \& Snyder, A. (1995). Effects of specific versus cross-training on running performance. European Journal of Applied Physiology and Occupational Physiology, 70(4), $367-372$. doi: $10.1007 / \mathrm{bf} 00865035$

Fox III, S.M., \& Naughton, J.P., (1972). Physical activity and the prevention of coronary heart disease. Preventive Medicine, 1(1-2), 92-120.

Herman, L., Foster, C., Maher, M.A., Mikat, R.P., \& Porcari, J.P. (2006). Validity and reliability of the session RPE method for monitoring exercise training intensity. South African Journal of Sports Medicine, 18(1), 14-17. doi: $10.17159 / 2078-516 x / 2006 / v 18 i 1 a 247$

Hopkins, W.G. (2008). A scale of magnitudes for effect statistics. Retrieved from www.sportsci.org/resource/stats/ effectmag.htlm

Hopkins, W.G., Marshall, S.W., Batterham, A.M., \& Hanin, J. (2009). Progressive statistics for studies in sports medicine and exercise science. Medicine and Science in Sports and Exercise, 41, 3-13. doi: doi.org/10.1249/ mss.0b013e31818cb278

Impellizzeri, F.M., Rampinini, E., Coutts, A.J., Sassi, A., \& Marcora, S.M. (2004). Use of RPE based training load in soccer. Medicine and Science in Sports and Exercise, 36(6), 1042-1047. doi: 10.1249/01.mss.0000128199.23901.2f

Lupo, C., Capranica, L., Cortis, C., Guidotti, F., Bianco, A., \& Tessitore, A. (2017a). Session-RPE for quantifying load of different youth taekwondo training sessions. Journal of Sports Medicine and Physical Fitness, 57(3),189194. doi: 10.5114/biolsport.2017.63381

Lupo, C., Capranica, L., \& Tessitore, A. (2014). The validity of session-RPE method for quantifying training load in water polo. International Journal of Sports Physiology and Performance, 9(4), 656-660. doi: 10.1123/ijspp.2013-0297

Lupo, C., Tessitore, A., Gasperi, L., \& Gómez, M.A. (2017b). Session-RPE for quantifying the load of different youth basketball training sessions. Biology of Sport, 34,11-17. doi: 10.5114/biolsport.2017.63381

Minganti, C., Capranica, L., Meeusen, R., \& Piacentini, M.F. (2011). The use of session-RPE method for quantifying training load in diving. International Journal of Sports Physiology and Performance, 6(3), 408-418. doi: 10.1123/ ijspp.6.3.408

Murphy, A.P., Duffield, R., Kellett, A., \& Reid, M. (2016). A comparison of the perceptual and technical demands of tennis training, simulated match play, and competitive tournaments. International Journal of Sports Physiology and Performance, 11(1), 40-47. doi: 10.1123/ijspp.2014-0464

Nikolaidis, P.T. (2015). Maximal heart rate in soccer players: Measured versus age-predicted. Biomedical Journal, 38 , 84-89. doi: 10.4103/2319-4170.131397

Pilaczyńska-Szcześniak, L., Lisiecki, D., Kasprzak, Z., Karolkiewicz, J., Nowak, A., Lewandowska, M., PilaczyńskaSzcześniak, L., \& Lisiecki, D. (2011). Effects of annual training cycle on the metabolic response to supra-maximal exercise test in beach volleyball players. Journal of Human Kinetics, 27, 80-94. doi: 10.2478/v10078-011-0007-z

Scott, T., Black, C., Quinn, J., \& Coutts, A.J. (2013). Validity and reliability of the session-RPE method for quantifying training in Australian football: A comparison of the CR10 and CR100 scales. Journal of Strength and Conditioning Research, 27, 270-276. doi: 10.1519/jsc.0b013e3182541d2e

Tanaka, H., Monahan, K.D., \& Seals, D.R. (2001). Age-predicted maximal heart rate revisited. Journal of the American College of Cardiology, 37, 153-156. doi: 10.1016/s0735-1097(00)01054-8

Wallace, L.K., Slattery, K.M., \& Coutts, A.J. (2009). The ecological validity and application of the session-RPE method for quantifying training loads in swimming. Journal of Strength and Conditioning Research, 23(1), 33-38. doi: $10.1519 /$ jsc.0b013e3181874512

Submitted: January 16, 2019

Accepted: January 7, 2020

Published Online First: April 6, 2020

Correspondence to:

Corrado Lupo, Ph.D.

Neuro Muscular Function Research Group,

School of Exercise and Sport (SUISM),

Department of Medical Sciences,

University of Turin, Italy

E-mail: corrado.lupo@unito.it 